\title{
Full mouth rehabilitation of the patient with severely worn dentition: a case report
}

\author{
Mi-Young Song, DDS, MSD, Ji-Man Park, DDS, MSD, Eun-Jin Park*, DDS, MMSc, PhD \\ Department of Prosthodontics, School of Medicine, Ewha Womans University, Seoul, Korea
}

\begin{abstract}
The severe wear of anterior teeth facilitates the loss of anterior guidance, which protects the posterior teeth from wear during excursive movement. The collapse of posterior teeth also results in the loss of normal occlusal plane and the reduction of the vertical dimension. This case report describes 77-year-old female, who had the loss of anterior guidance, the severe wear of dentition, and the reduction of the vertical dimension. Occlusal overlay splint was used after the decision of increasing vertical dimension by anatomical landmark, facial and physiologic measurement. Once the compatibility of the new vertical dimension had been confirmed, interim fixed restoration and the permanent reconstruction was initiated. This case reports that a satisfactory clinical result was achieved by restoring the vertical dimension with an improvement in esthetics and function. [J Adv Prosthodont 2010;2:106-10]
\end{abstract}

KEY WORDS. Tooth wear, Vertical dimension of occlusion, Occlusal overlay splint

\section{INTRODUCTION}

The gradual wear of the occlusal surfaces of teeth is a normal process during the lifetime of a patient. However, excessive occlusal wear can result in pulpal pathology, occlusal disharmony, impaired function, and esthetic disfigurement. ${ }^{1}$ Tooth wear can be classified as attrition, abrasion, and erosion depending on its cause. A differential diagnosis is not always possible because, in many situations, there exists a combination of these processes. ${ }^{2}$ Therefore, it is important to identify the factors that contribute to excessive wear and to evaluate alteration of the VDO caused by the worn dentition. ${ }^{3}$

In many cases, the vertical dimension of occlusion (VDO) is maintained by tooth eruption and alveolar bone growth. As teeth are worn, the alveolar bone undergoes an adaptive process and compensates for the loss of tooth structure to maintain the VDO. Therefore, VDO should be conservative and should not be changed without careful approach. ${ }^{4,5}$ Especially, increasing the VDO in bruxers puts a severe overload on the teeth and often results in the destruction of the restorations or teeth themselves. ${ }^{4}$

However, the rehabilitation of the severely worn dentition is challenging when the space for restoration is not sufficient. In 1975, Dahl et al. ${ }^{6}$ reported the use of a removable cobalt-chromi- um anterior occlusal device to an 18-year-old patient with advanced localized attrition to generate interocclusal space for subsequent restoration. The tooth movement involving a combination of orthodontic anterior teeth intrusion and eruption of the posterior teeth occurred. And long-term observations of this treatment were reported that the vertical relations were practically stable. ${ }^{7.8}$ Nowadays this technique is replaced by using the adhesive resin ${ }^{9,10}$ or an overlay splint ${ }^{5,11,12}$ instead of a cobalt-chromium device.

Management of worn dentition using fixed or removable prostheses is complex and among the most difficult cases to restore. Assessment of the vertical dimension is important for the management, and careful comprehensive treatment plan is required for each individual case. Articulated study casts and diagnostic wax-up can provide important information which is helpful for the evaluation of treatment options. Tolerance of changes to vertical dimension of occlusion is usually confirmed with the clinical evaluation of the patient having a diagnostic splint or provisional prosthesis. ${ }^{13}$

This clinical report describes the treatment of a patient who was clinically monitored to evaluate the adaptation to the removable occlusal overlay splint during a 1 month trial period and the provisional restorations for 3 months..$^{14,15}$
Corresponding author: Eun-Jin Park

Department of Prosthodontics, School of Medicine, Ewha Womans University

911-1, Mok-6-dong, Yangchun-gu, Seoul, 158-710, Korea

Tel. 8222650 5042: e-mail, prosth@ewha.ac.kr

Received August 12, 2010 / Last Revison August 30, 2010 / Accepted September 3, 2010 (c) 2010 The Korean Academy of Prosthodontics

This is an Open Access article distributed under the terms of the Creative Commons Attribution Non-Commercial License (http://creativecommons.org/licenses/bync/3.0) which permits unrestricted non-commercial use, distribution, and reproduction in any medium, provided the original work is properly cited. 


\section{CASE REPORT}

A 77-year-old woman was referred for the treatment of her severely worn dentition. Her chief complaint was that she could not eat anything because her teeth were worn too much. The patient had anticoagulant and analgesic agent due to hypertension and idiopathic headache. Intraoral examination revealed a generalized loss of dental substance that was greater in the maxillary left incisors and the mandibular right incisors. Maxillary left canine and mandibular right canine were worn to the gingival level, and had got root canal treatments (Fig. 1). The anterior teeth had sharp enamel edges, dentinal craters, and attritional wear due to the loss of posterior support. The mandibular posterior teeth were missing, but she said that she did not use her old removable partial denture (RPD) as it was not comfortable. The mandibular RPD lost its retention and support because the regions that functioned as the rest and retentive undercut of abutment teeth had been fractured. The facial type of patient was square and her lip seemed to be under strong tension. The patient did not have temporomandibular disorder history and soreness of the mastication muscles, but the discrepancy between centric occlusion (CO) and maximum intercuspal position (MIP) was found when she was guided to $\mathrm{CR}$ with bimanual technique. The transcranial view was taken to determine whether a temporomandibular problem exists. The right mandibular condyle was flatter than the left one, but any specific disorder was not found (Fig. 2).

To determine whether VDO had been altered, the following aspects were investigated: $:^{1,5,11}$

1. Loss of posterior support: mandibular posterior teeth were missing, and the patient did not use the mandibular partial denture. Posterior collapse resulted in excessive wear and fracture of anterior teeth.

2. History of wear: Physiologic wear can be compensated by tooth eruption in general, but the accelerated wear may exceed the rate of eruption. The patient liked vegetables and acidic fruits. Her favorite food was tough and fibrous. In addition, maxillary posterior base metal pros- theses might accelerate the wear of mandibular acrylic resin teeth and unbalance of wear rate.

3. Phonetic evaluation: If the distance between the incisal edge of the mandibular incisors and lingual surface of the maxillary incisors is about $1 \mathrm{~mm}$, it makes normal /s/ sound. The patient's increased space altered /s/ sound to / $\int /$.

4. Interocclusal rest space: The patient' $s$ interocclusal rest space that was measured between nose tip and chin tip was 5 $6 \mathrm{~mm}$ that was greater than the normal value, $2-4 \mathrm{~mm}$.

5. Facial appearance: Wrinkles and drooping commissures around mouth were observed.

The possible causes of patient's worn dentition that might include posterior interferences, parafunction, eating habit, and dental ignorance were explained to the patient. And the options of treatment plan were restoring mandibular edentulous posterior region with implants or removable partial denture, full mouth rehabilitation with metal ceramic restoration with or without crown lengthening procedure. The patient was scared of implant surgery, so the option of implant installation was excluded. Also the patient did not want to get multiple crown lengthening procedures and endodontic treatments to restore the worn teeth in the insufficient space. As there was clinical evaluation of reduced VDO, full mouth rehabilitation with

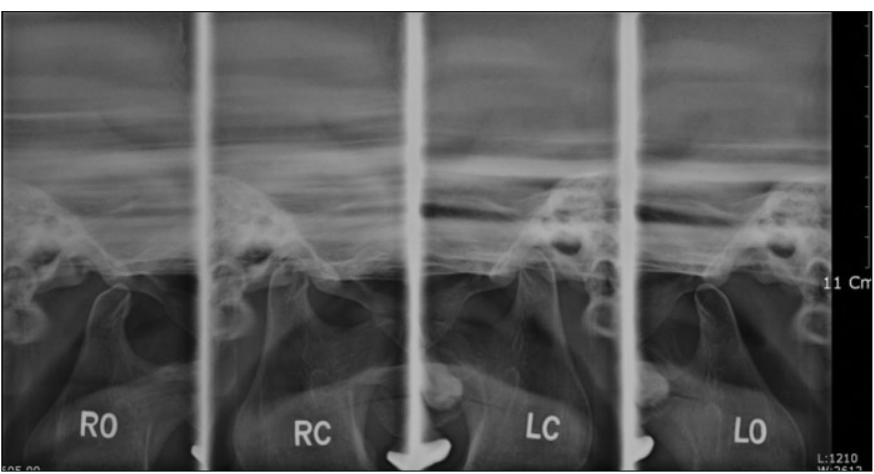

Fig. 2. Transcranial view of TMJ. Disfigurement or limitation of movement of TMJ was not detected.
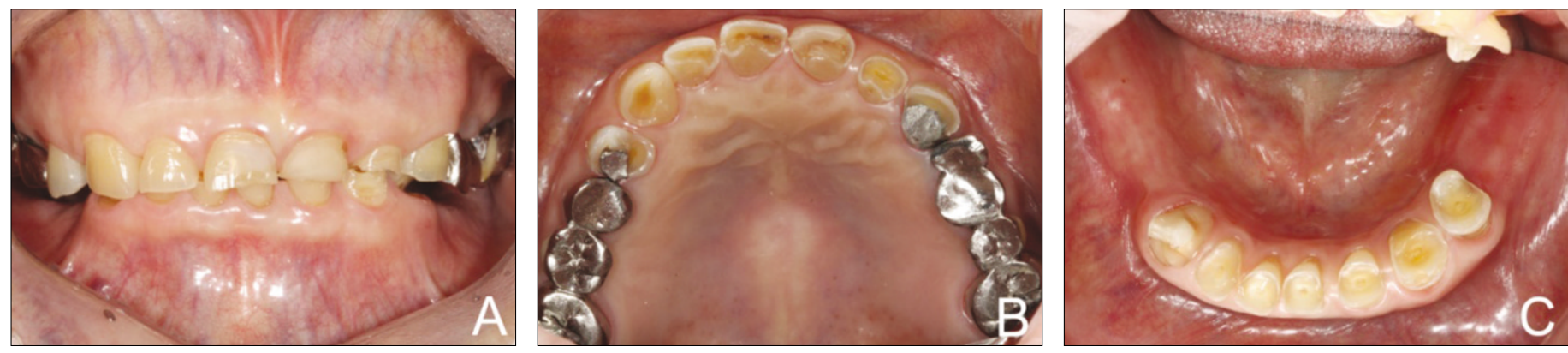

Fig. 1. Initial oral examination. A: Frontal view. Severely worn dentition and loss of posterior support were shown. B: Maxillary occlusal view. The palatal surface of maxilliary left canine was worn to the gingival level and got root canal treatment. C: Mandibular occlusal view. The severe attrition and abfraction on anterior teeth were shown. 
increasing VDO was planned. Only severely worn teeth which were maxillary left canine and mandibular right canine were undergone crown lengthening procedures to obtain a sufficient clinical crown length and ferrule effect. ${ }^{16}$

The patient's casts were mounted on a semi-adjustable articulator (Hanau ${ }^{\mathrm{TM}}$ Modular Articulator; Whip Mix Corp., Louisville, USA) using a face-bow record and an interocclusal record that was made with the aid of a Lucia jig and polyvinylsiloxane occlusal registration material (EXABITE II; GC Corp., Tokyo, Japan). The new VDO was set by $5 \mathrm{~mm}$ increase in the incisal guidance pin of the articulator. Because the patient' $\mathrm{s}$ interocclusal rest space was $2-3 \mathrm{~mm}$ larger on the premolar area than normal distance, the actual increase were determined $3 \mathrm{~mm}$ in the anterior teeth and $1-2 \mathrm{~mm}$ in the posterior teeth. The splint was designed to offer bilateral contacts of all posterior teeth in centric relation and guides of the anterior teeth in excursive movement (Fig. 3). The anterior guidance disoccluded the posterior teeth in all jaw position except centric relation.

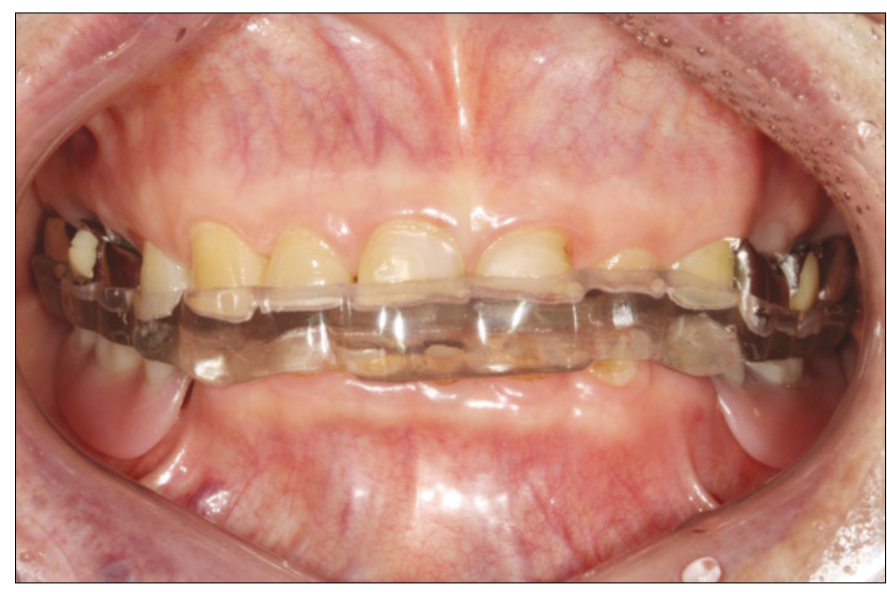

Fig. 3. Occlusal overlay splint was delivered and monitored for 1 month to evaluate patient's adaptation to the new VDO.

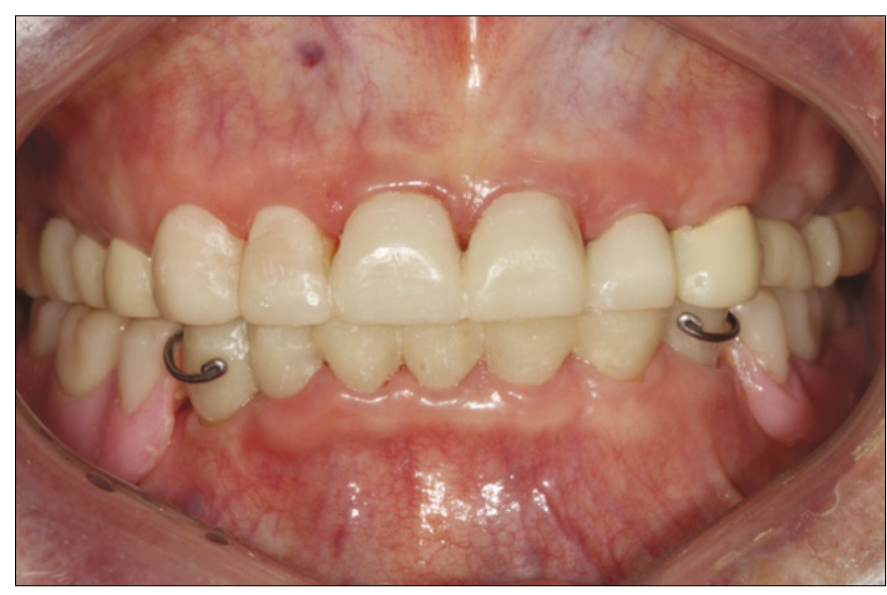

Fig. 5. Provisional restorations were placed after trial period of removable occlusal overlay splint.
The adaptation of patient to the increased VDO was evaluated during 1-month trial period. No muscle tenderness and temporomandibular discomfort was found. The method of increasing VDO with the splint was used to determine desirable VDO of the fixed interim prostheses. After taking CR record using Lucia jig and wax-rim, diagnostic wax-up was performed (Fig. 4). Autopolymerizing acrylic resin (ALIKE' ${ }^{\mathrm{TM}}$; GC America, ALSIP, USA) provisional crowns were fabricated using a vacuum formed matrix (Drufolen H; Dreve Dentamid $\mathrm{GmbH}$, Unna, Germany) that was produced from the diagnostic wax-up, and mandibular provisional RPD was made to fit provisional crowns (Fig. 5). The provisional fixed restorations were cemented with temporary cement (FREEGENOL TEMPORARY PACK; GC Corp., Tokyo, Japan), and the patient's adaptation was monitored.

For three months, interim restorations were adjusted, and used as a guide for the definitive oral rehabilitation. During this period, the patient's condition and functions, such as muscle tenderness, discomfort of TMJ, mastication, range of the

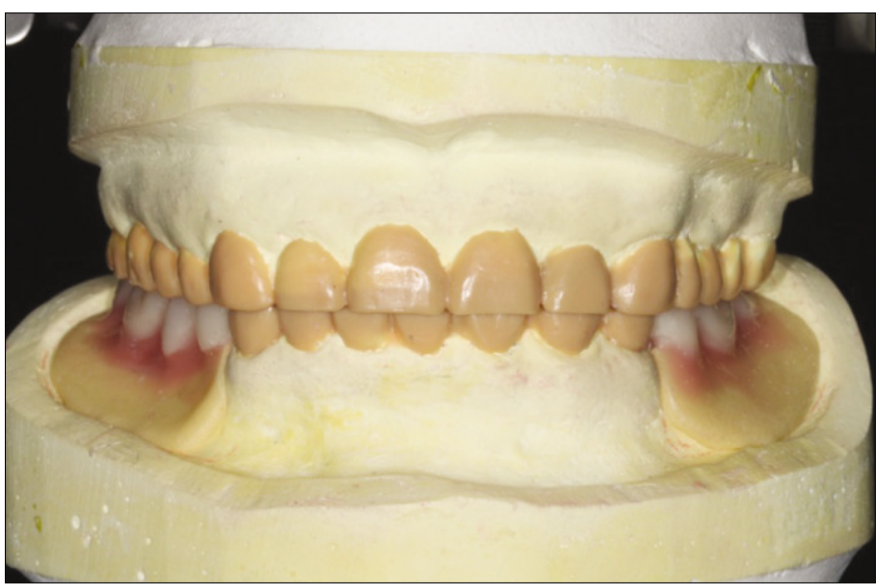

Fig. 4. Diagnostic wax-up was done on the new VDO that was obtained by adjustment using the overlay occlusal splint.

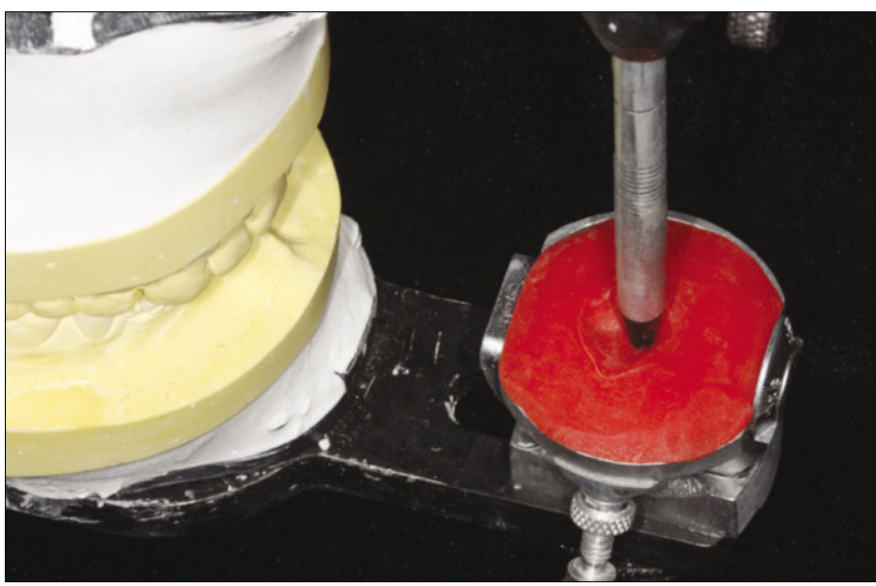

Fig. 6. Customized anterior guide was made utilizing the duplicated provisional restoration casts. 

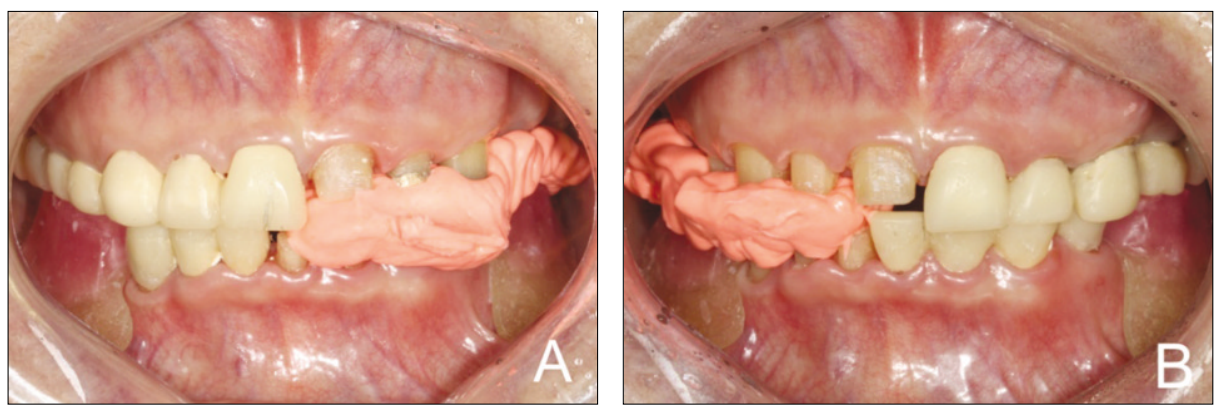

Fig. 7. Bite registration was taken using provisional restoration and bite registration material by half and half. A: Left bite registration, B: Right bite registration.
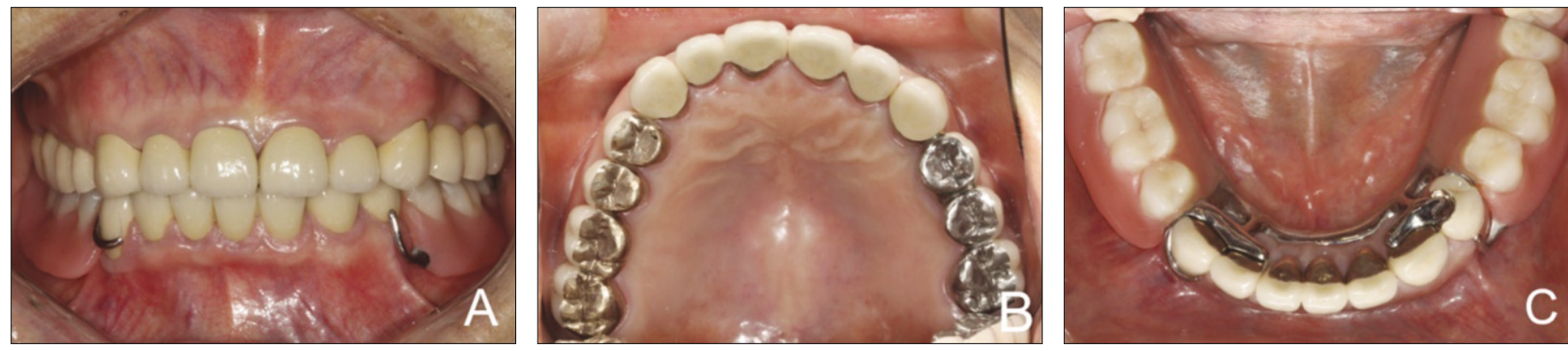

Fig. 8. Definitive restoration was delivered. A: frontal view, B: maxilliary occlusal view, C: mandibular occlusal view.

mandibular movements, swallowing, and speech, were evaluated. Improvement in mastication, speech, and facial esthetics confirmed the patient s tolerance to the new mandibular position with the restored VDO. The anterior guidance and posterior disclusion on excursive movement were established. Adjusted occlusion was transferred to customized anterior guide table, which was made with acrylic resin (PATTERN RESIN; GC Corp, Tokyo, Japan $)^{17}$ (Fig. 6).

Final preparation was performed, and definitive impressions were made with polyvinylsiloxane impression material (Extrude; Kerr Corp., Romulus, Germany). Bite registration was taken using provisional crown and occlusal registration material (StoneBite; Dreve Dentamid GmbH, Unna, Germany) by half and half (Fig. 7). Porcelain fused to metal restorations were made using customized anterior guide table and cemented with resin modified glass ionomer cement (FujiCEM; GC America, Alsip, USA). Because the patient's anterior guidance table was used in the production of definitie restoration, the amount of occlusal adjustment on the lingual surface of maxillary anterior teeth was minimal. Individual tray with polyvinylsiloxane (EXAMIXFINE; GC Corp., Tokyo, Japan) was used for the impression of mandibular RPD. The impression on posterior alveolar ridge was taken once more with the individual tray which is attached to the RPD framework, and the altered cast was made. After the adaptation of RPD framework and the trial of wax denture were done, the definitive mandibular RPD was fabricated and delivered with minor occlusal adjustment (Fig. 8). The prostheses were designed using mutually protected occlusion. The anterior teeth protected the posterior teeth from excursive force and wear, and posterior teeth supported the bite force. Oral hygiene instruction and regular check-up were administered.

\section{DISCUSSION}

In 1984, Turner $^{1}$ classified the treatment of a severely worn dentition by the amount of the loss of VDO and available space to restore. His classification and conventional treatment, which includes raising VDO with multiple crown-lengthening procedures, have been widely used up to present. However, the etiology of tooth wear is multifactorial, and clinical controlled trials of restorative and prosthodontic approaches are limited in quantity and quality. In addition, lack of evidence regarding the long-term outcomes of treatment methods and materials cause difficulty in clinical decision-making. ${ }^{18}$ Because of these unclear guidelines, adhesive strategy, that is more conservative and reversible, is increasing. ${ }^{9,10,18}$ Nonetheless, the composite resin restoration could not be used for the patient in this case. The remaining tooth structures were too small to have sufficient retention of composite resin, and the surveyed crowns to support RPD were necessary. Therefore, the conventional treatment modality that includes a trial overlay splint, provisional restoration, careful monitoring, and definitive prosthesis, was chosen. 
In previous literature, the wearing time of overlay splint and provisional crown is various. The trial period of overlay prostheses which are reversible and conservative is between 3 weeks and 5 months, and that of intensive fixed provisional prostheses is $2-6$ months..$^{1,5,9,11,19}$ In this case, the patient was carefully monitored for 1 month to evaluate the adaptation to the removable occlusal overlay splints. ${ }^{15}$ Also the patient' $\mathrm{s}$ adaptation to the provisional restoration was monitored for 3 months..$^{14}$ The trial period is relatively shorter than the other case report, but discomfort, wear, and muscle fatigue were not observed during that period. The increase of VDO was determined not by standardized esthetic golden proportion of anterior teeth but by patient's physiologic factor like interocclusal rest space and speech. If the increase of VDO was decided arbitrarily without close evaluation, multiple complications would happen and longer treatment period might be needed. Depending on the patient's situation and adaptation ability, the interim period can be modified, and the careful evaluation and monitoring may shorten the overall treatment duration.

The rehabilitation using restoration of anterior crowns and RPD providing posterior support is affordable and common for many patients who require the treatment of teeth wear because of reasons of economics and tradition..$^{18}$ However, the restored anterior teeth can be easily exposed to excessive occlusal loads if the patient does not wear the RPD or resorption of residual ridge proceeds. Because the compliance of patients in wearing free-end saddle dentures has been shown to be poor, ${ }^{20}$ the education on wearing RPD is necessary. Regular check-up for the occlusal adjustment and RPD fitting is essential.

\section{CONCLUSION}

In this clinical report, raising vertical dimension of occlusion using removable occlusal overlay splint and following fixed provisional based on accurate diagnosis showed successful full mouth rehabilitation for severely worn down dentition.

\section{REFERENCES}

1. Turner KA, Missirlian DM. Restoration of the extremely worn dentition. J Prosthet Dent 1984;52:467-74.

2. Smith BG. Toothwear: aetiology and diagnosis. Dent Update 1989;16:204-12.

3. Prasad S, Kuracina J, Monaco EA Jr. Altering occlusal vertical dimension provisionally with base metal onlays: a clinical report. J Prosthet Dent 2008;100:338-42.

4. Dawson PE. Functional Occlusion - From TMJ to smile design. $1^{\text {st }}$ ed. New York; Elsevier Inc.; 2008. p. 430-52.

5. Jahangiri L, Jang S. Onlay partial denture technique for assessment of adequate occlusal vertical dimension: a clinical report. J Prosthet Dent 2002;87:1-4.

6. Dahl BL, Krogstad O, Karlsen K. An alternative treatment in cases with advanced localized attrition. J Oral Rehabil 1975;2:20914.

7. Dahl BL, Krogstad O. Long-term observations of an increased occlusal face height obtained by a combined orthodontic/prosthetic approach. J Oral Rehabil 1985;12:173-6.

8. Dahl BL. The face height in adult dentate humans. A discussion of physiological and prosthodontic principles illustrated through a case report. J Oral Rehabil 1995;22:565-9.

9. Hemmings KW, Darbar UR, Vaughan S. Tooth wear treated with direct composite restorations at an increased vertical dimension: results at 30 months. J Prosthet Dent 2000;83:287-93.

10. Darbar UR, Hemmings KW. Treatment of localized anterior toothwear with composite restorations at an increased occlusal vertical dimension. Dent Update 1997;24:72-5.

11. Sato S, Hotta TH, Pedrazzi V. Removable occlusal overlay splint in the management of tooth wear: a clinical report. J Prosthet Dent 2000;83:392-5.

12. Windchy AM, Morris JC. An alternative treatment with the overlay removable partial denture: a clinical report. J Prosthet Dent 1998;79:249-53.

13. Hemmings KW, Howlett JA, Woodley NJ, Griffiths BM. Partial dentures for patients with advanced tooth wear. Dent Update 1995;22:52-9.

14. Yunus N, Abdullah H, Hanapiah F. The use of implants in the occlusal rehabilitation of a partially edentulous patient: a clinical report. J Prosthet Dent 2001;85:540-3.

15. Ganddini MR, Al-Mardini M, Graser GN, Almog D. Maxillary and mandibular overlay removable partial dentures for the restoration of worn teeth. J Prosthet Dent 2004;91:210-4.

16. Hempton TJ, Dominici JT. Contemporary crown-lengthening therapy: a review. J Am Dent Assoc 2010;141:647-55.

17. Hoyle DE. Fabrication of a customized anterior guide table. J Prosthet Dent 1982;48:490-1.

18. Johansson A, Johansson AK, Omar R, Carlsson GE. Rehabilitation of the worn dentition. J Oral Rehabil 2008;35:548-66.

19. Brown KE. Reconstruction considerations for severe dental attrition. J Prosthet Dent 1980;44:384-8.

20. Witter DJ, Van Elteren P, Käyser AF, Van Rossum GM. Oral comfort in shortened dental arches. J Oral Rehabil 1990;17:137-43. 